\title{
Environmental impact reduction of commercial aircraft around airports. Less noise and less fuel consumption
}

\author{
Salah Khardi
}

Received: 9 March 2012 / Accepted: 29 May 2013 / Published online: 9 July 2013

(C) The Author(s) 2013. This article is published with open access at SpringerLink.com

\begin{abstract}
Introduction Flight path optimization is designed for minimizing environmental impacts of aircraft around airports during approaches. The main objective of this paper is to develop a model of optimal flight paths taking into account jet noise, fuel consumption, constraints and extreme operational limits of the aircraft in approach.

Materials and methods Optimal control problem combining aircraft flight paths, noise and fuel consumption is modeled and solved. Outputs, algorithm efficiency and their ability to be interfaced with the in-flight management system, respecting airspace system regulation constraints, are analyzed. Measurements of aircraft noise are carried out around Saint-Exupery Lyon International Airport for one year, and INM simulations are performed.

Results and conclusion A two-segment approach is considered as an optimal trajectory. Aircraft alignment on the runway axis with a slope between $3^{\circ}$ and $4.5^{\circ}$ during the last approach segment is obtained and the descent rate is about $1060 \mathrm{ft} / \mathrm{mn}$. This particularly characterizes the continuous descent approach having the potential to reduce noise emission by $-4 \mathrm{~dB}$ and fuel consumption by $-20 \%$ to $-10 \%$ during the approach. Because of the suggested trajectory, optimized noise levels are less than the measured and INM values given by the empirical trajectory. Optimal trajectory consumes less than the standard one; and it can be integrated in the aircraft FMS and the autopilot system. This is one of
\end{abstract}

\footnotetext{
S. Khardi $(\bowtie)$

Transport and Environment Laboratory, The French Institute

of Science and Technology for Transport,

Development and Networks, IFSTTAR,

25 avenue F. Mitterrand, 69675 Bron, France

e-mail: salah.khardi@ifsttar.fr
}

the promising objectives of this research. To conclude, environmental impacts and fuel consumption are reduced by the use of aircraft trajectory optimization during arrivals.

Keywords Environment - Impacts · Aircraft · Flight path · Noise reduction $\cdot$ Fuel consumption

\section{Introduction}

The airspace system is becoming increasingly congested as the number of aircraft operations grows to meet passenger and goods demands. The bulk of traffic contributes to airport traffic saturation. Due to this increase, populations living near airports, as well as the environment, are impacted by commercial aircraft.

Aircraft noise is considered to be one of the most significant environmental concerns in the local communities of modern cities, affecting people living near airports. This is supported by evidence that aircraft noise exposure is associated with reduced well-being, lower self-reported quality of life and higher levels of self-reported stress, anxiety, depression and psychological morbidity. Significant work has been done in the area of noise effects reflecting different aspects of annoyance and human health considerations. This is a critical issue that affects the sustainability of commercial aviation. Different solutions have been attempted to control aircraft noise at airports. Nevertheless, noise levels in the vicinity of airports, in particular under the take-off and landing flight paths remain high and disrupt the quality of life of local residents. This is considered today to be one of the most significant environmental concerns affecting population and the environment [1].

The historical trend in aircraft noise has shown a reduction of approximately $20 \mathrm{~dB}$ since the 1960 s largely due to 
the adoption of high bypass turbofans and more effective lining materials. Reductions since the mid-eighties have not been as dramatic. The point seems to have been reached where future improvements through technological advances will be possible only by significantly trading off operating costs for environmental performance.

In addition, a number of major European airports have reached their environmental capacity before having made full use of their runway and terminal infrastructures. Thus, the significant environmental challenges of the Advisory Council for Aeronautics Research in Europe have been sub-divided into four goals:

- To reduce fuel consumption and $\mathrm{CO} 2$ emissions by $50 \%$;

- To reduce perceived external noise by $50 \%$;

- To reduce NOx by $80 \%$;

- To make substantial progress in reducing the environmental impact of the manufacture, maintenance and disposal of aircraft and related products.

Contributors to noise reduction should encompass technology related elements such as the Quiet Aircraft and Rotorcraft of the future as well as further actions aimed at establishing efficient Environmental Practices by way of Noise Abatement Procedures and Management of Noise Impact.

The control of noise around airports is a complex matter because it depends on many different factors, and many different issues are involved. Significant research is currently being undertaken with the goal of reducing aircraft noise. Existing projects have already identified a number of promising procedures and methods that could be applied to achieve an harmonized approach within the EU and further steps should go a step further, by developing enabling technologies, tools and methods to ensure the safe, efficient and economic operation of new noise abatement procedures. The suggested research activities for aircraft should cover both:

- The definition of noise abatement flight procedures through flight test demonstrations, taking account of the safety and of operational constraints.

- The development of pilot aids to ease the different operations through specific control laws using specific guidance system (as for instance GPS or D-GPS).

- The development of real time noise footprint assessment to be used for in-flight demonstration and for piloted simulation to elaborate control strategies.

These activities generally fall into one of the three categories: noise source modifications (e.g. retrofitting engine nacelles with sound absorption material), land use regulations (e.g. zoning land near an airport as nonresidential), and operational controls (e.g. power cutbacks during operations; this is considered impractical because the pilot workloads and flight safety decrease). Modification of the ends of flap sections and other aerodynamic treatment will have some success; but there is no great optimism. It is necessary to examine the potential for modified approach procedures that can benefit both airlines and the communities. The International Civil Aviation Organization (ICAO) has recognized the potential benefits of using advanced flight guidance technology to reduce the impact of aircraft noise [2, 23, 26, 42]. ICAO charged its Noise Abatement Operating Measures Subgroup (NAOMSG) with the following tasks:

1. Describe effective existing noise abatement operational procedures and strategies.

2. Evaluate the critical components of aircraft flight procedures that can minimize source noise emissions and community exposure.

3. Identify emerging and future airport systems technologies in the fields of flight management, ATC and airport capacity which could also serve to minimize community noise exposure.

4. Conceive new operating procedures to reduce community noise exposure taking into account the emerging and future technologies identified in 3 .

It should be remembered that the assessment of the appropriate noise abatement procedure to use needs to consider several coupled factors:

- aircraft performance and trajectory;

- noise generated by the aircraft;

- population distribution and density;

- flight safety and pilot acceptance;

- guidance and navigation requirements;

- local atmospheric conditions.

Advanced flight guidance technologies, which are currently in use, such as Area Navigation (RNAV) utilizing the Global Positioning System (GPS), offer the potential to reduce the impact of aircraft noise in communities surrounding airports by enabling more flexible approach and departure procedures that reduce noise exposure to the most sensitive areas.

A balanced approach to environmental protection has been developed under ICAO and it includes protection from excessive aircraft noise first. There are several procedures that have been implemented in aircraft operation world-wide:

1) low-noise take-off and approach flight procedures;

2) optimal distribution of the aircraft among the routes;

3) flight route optimization in the airport vicinity. In combination, the aircraft, airport, and the community form a closed system. If flight procedures are the means of 
operating that system, then the noise abatement procedure represents a way of operating the system with lower noise impact.

The Committee on Aviation Environmental Protection (CAEP) presented the following described Balanced Approach on aircraft noise management around airports [2, $17,26,41]$.

Concept of a balanced approach An aircraft is a major investment, with a useful economic life of 25 years or more. Operation of an aircraft includes airframe and engine performance. The performance of an aircraft must address the overriding issue of safety, as well as mission or performance efficiencies, economics, and environmental objectives.

Across the various organizations, the EU, the United States, and ICAO the fundamental approach to analysis and management or control of aircraft noise is similar. All recommend a "balanced" approach that includes at least four ingredients: noise control at the source (the aircraft), land-use planning around airports, noise abatement operating procedures for aircraft, and restrictions on operations. In addition to these four categories, in the United States, the FAA includes airport layout and ICAO recognizes noise charges. To pursue the balanced approach, specific tools are required. First, metrics or indicators of noise must be identified, noise effects or impacts in relation to the indicators need to be defined, and a method for computing the values of the metrics in communities around airports is required. The ICAO Assembly endorsed the concept of a balanced approach to aircraft noise management. Within ICAO, CAEP developed the requisite guidance material. Four distinct elements have to be considered and analyzed: Airspace, airfield, terminal, ground access.

Most of the world major airports have operational constraints or capacity limits based upon noise. But the future potential growths of air traffic imply that emission sources in the future will increase in importance. The study of integrated airport impact shows that it is necessary to introduce the concept of airport traffic (operational) capacity according to environmental safety conditions. Evaluation of an airport impact on surrounding environment could be realized by defining environmental capacity of an airport. It means reduction of an airport's capacity so as to ensure that airport environmental performances comply with the environmental rules. Operational capacity of an airport can be measured as the number of runway-taxiways slots, the terminal capacity or capacity of the apron areas. It is limited only by means of flight safety. The economic capacity of an airport can be measured as the maximum number of passengers or aircraft, which can be accommodated on a particular day with a given amount of infrastructure under given economic conditions. In a short term, airport service load during peak and off-peak period determine these conditions. In long term, the availability of investments for airport expansion principally determines the economic conditions.

The impacts of the airport operation upon the local environment are a major issue, which will affect both the capacity and the potential for future growth. This concept of environmental capacity as it applies to airports can be approached in at least two ways: the first is that an airport operational capacity is less than the total sum of the individual environmental mitigation measure already in place at that airport. The second is or could ever lead to an environmentally optimal solution. It is necessary to identify and separate short term concerns which mainly affect quality of life (e.g. aircraft noise) from long term issues which mainly affect the assimilative capacity of the environment to cope with what we are throwing at it (e.g. pollution and global warming). In addition, it is necessary to assess the viability of the environmental mitigation measures that are in the airport territory and in the vicinity. For example, many major airports have long-established night flight restrictions whose aim has been to protect local communities from excessive exposure from aircraft noise. From an environmental capacity perspective, such restrictions may be seen as short-run, quality of life issue and a successful mitigation measure but with potentially more serious long term environmental consequences.

Thus, CAEP identified noise problem and discussed the measures for its reduction and control. Among four elements of a Balanced Approach, current investigations deal with Noise Abatement Procedures (NAP), which according to ICAO's policies, enable the reduction of noise during aircraft operations. New flight path development is a solution which should contribute to a decrease of aircraft noise annoyance. It will also meet objectives 2020 of the Advisory Council for Aeronautics Research in Europe (ACARE). CAEP, OACI and ACARE reported that flight path optimization can provide a sizable decrease in noise impact depending not only on the population distribution, but on the types and the numbers of aircraft operations.

This environmental problem can only be solved within the framework of a balanced global vision for a sustainable air transport involving new technology engines and fuselages, breakthrough technologies, the design of new procedures and flight paths, airspace management, new regulation rules and certification. Thus, technological developments, airspace management, operational improvement and system efficiency should be considered as environmental innovations. There is no justification that air transport will not continue to progress without decreasing its environmental impacts [2]. The applied procedures are not optimized but are generic in nature. New flight path 
optimization, associated with new aircraft design and engines, is a solution which should contribute to a decrease in aircraft annoyances. Noise abatement procedures are considered as a necessary measure for a balanced approach of noise control around airports and for fuel consumption saving. Any system, which defines the correct features of the optimized flight paths for aircraft in specific conditions, would be useful for environmental impact control. Developments cannot be carried out without improvement modeling. This consists of developing efficient processing tools which allow in-flight diagnosis and control in real-time taking into account the FMS (flight management system) functionalities and the AMS (airspace management system) updates. Flight path optimization is an innovative solution in the short run, making a significant contribution to the reduction of commercial aircraft impact on the environment possible.

This paper presents a dynamic method providing optimal flight paths which minimize aircraft impacts and fuel consumption. This is an optimal control problem to be solved. The main features of the suggested method are its effectiveness and its resolution speed. It shows a significant potential of its own integration in the avionic systems. That is why in this paper we have solved the flight path optimization problem with the aim of confirming its advantages, reliability and features. We have suggested an optimization method for solving a model governed by an $O D E$ system $[3,4]$ providing the best flight path suitable for noise and fuel consumption reduction. The cost function of this model describes aircraft noise and fuel consumption $[5,6]$. The ODE depends on the flight dynamics of the aircraft, and considers flight safety and stability requirements. Numerical methods which solve the ODE fall into several categories [7-11] which depend on the case study. Thus, it is necessary to choose, to improve or develop a new method. In this context, this paper gives numerical considerations and algorithms for solving the control problem stressing the computing times with the aim of finding the best aircraft approach able to reduce noise and favoring economization of fuel consumption. The applied approach has been used to reduce the $(O C P)$ to a finite-dimensional nonlinear program which is solved by a standard nonlinear programming solver.

Optimality conditions, given by Pontryagin's principle, have been discretized. A combination of the AMPL model (A Modeling Language for Mathematical Programming) [12] and an NLP solver [13-15] has been performed for calculations. In-depth details have been described in previous papers [16-19]. Technically, we analyze the processing outputs and algorithm efficiency and their ability to be interfaced with the in-flight management system respecting airspace system regulation constraints. This integration could compensate both the growth in air traffic and the encroachment of airport-neighboring communities.
This paper presents an introduction giving the optimal control problem and resolution, numerical results followed by an experimental measurements analysis and a conclusion. Measurements of aircraft noise, recorded under the flight path close to Saint-Exupery Lyon International Airport, are given. To validate the optimization method, the measured noise levels were compared to noise values obtained by INM for standard trajectories and by optimization method of flight path.

\section{Optimal control problem and resolution}

We present in this section a summary of the optimal control problem to be solved which is described in previous papers [16-19]. The system of differential equations commonly employed in aircraft trajectory analysis is the following sixdimensional system (ED) derived at the center of mass of the aircraft $[16,20]$ :

$$
\begin{aligned}
& \left(E D_{1}\right) \quad\left\{\begin{array}{l}
\dot{V}=g\left(\frac{T \cos \alpha-D}{m g}-\sin \gamma\right) \\
\dot{\gamma}=\frac{1}{m V}((T \sin \alpha+L) \cos \mu-m g \cos \gamma) \\
\dot{\chi}=\frac{(T \sin \alpha+L) \sin \mu}{m V \cos \gamma}
\end{array}\right. \\
& \left(E D_{2}\right) \quad\left\{\begin{array}{l}
\dot{x}=V \cos \gamma \cos \chi \\
\dot{y}=V \cos \gamma \sin \chi \\
\dot{h}=V \sin \gamma
\end{array}\right.
\end{aligned}
$$

where $V, \gamma, \chi, \alpha$ and $\mu$ are respectively the speed, the angle of descent, the yaw angle, the angle of attack and the roll angle. $(x, y, h)$ is the position of the aircraft. The variables $T, D, L, m$ and $g$ are respectively the engine thrust, the drag force, the lift force, the aircraft mass and the aircraft weight acceleration given in previous papers $[16,19-21]$. (ED) can be written in the following matrix form $\dot{z}(t)=$ $f(z(t), u(t))$, where:

$$
\begin{aligned}
& z:\left[t_{0}, t_{f}\right] \mapsto \mathbb{R}^{6} \\
& \quad t \mapsto z(t)=[V(t), \gamma(t), \chi(t), x(t), y(t), h(t)] \\
& \quad \text { are the state variables } \\
& \begin{array}{c}
u:\left[t_{0}, t_{f}\right] \\
\quad t \mapsto \mathbb{R}^{3}
\end{array} \\
& \text { and } t_{0} \text { and } t_{f} \text { are the initial and final times. }
\end{aligned}
$$

Along the flight path, we have to respect parameter limits related to the flight safety and the operational modes of the aircraft. Aircraft modeling continues to meet the increased demands associated with aviation and airport expansion. Aircraft noise footprints are commonly used for forecasting the impact of new developments, quantifying the noise 
trends around airports and evaluating new tools. Thus, aircraft models have become more sophisticated and their validation complex. A number of them are entirely based on empirical data. Because of this complexity, such models are not characterized by a given analytical form describing noise at reception points on the ground. This paper uses the basic principles of aircraft noise modeling. The cost function may be chosen as any of the usual aircraft noise indices, which describes the effective noise level of the aircraft noise event $[22,23]$. This study is limited to minimize the maximum noise level using a semi-empirical model of jet noise [5, 6, 24-26]. The cost function is expressed in the following form:

$$
\begin{aligned}
& J: \mathcal{C}^{1}\left(\left[t_{0}, t_{f}\right], R^{6}\right) \times \mathcal{C}^{1}\left(\left[t_{0}, t_{f}\right], \mathbb{R}^{4}\right) \mapsto R \\
& J(X(t), U(t))=\int_{t_{0}}^{t_{f}}\left(\ell(X(t), U(t))+\phi\left(X(t), t_{f}-t_{0}\right)\right) d t
\end{aligned}
$$

where $J$ is the criterion which optimizes noise levels completed by the cost describing fuel consumption $\phi$. The cost function, related to maximum emitted noise and fuel consumption function, can be written in the following integral form:

$$
\begin{aligned}
& J: \mathcal{C}^{1}\left(\left[t_{0}, t_{f}\right], \mathbb{R}^{6}\right) \times \mathcal{C}^{1}\left(\left[t_{0}, t_{f}\right], \mathbb{R}^{3}\right) \mapsto \mathbb{R} \\
& J(z(t), u(t))=\int_{t_{0}}^{t_{f}}\left(\ell(z(t), u(t))+\phi\left(z(t),\left(t_{f}-t_{0}\right)\right)\right) d t
\end{aligned}
$$

where $J$ is the criterion to be optimized. Finding an optimal trajectory can be stated as an optimal control problem as follows $\left(t_{0}=0\right)$ :

$$
(O C P) \quad\left\{\begin{array}{l}
\min J(z, u)=\int_{0}^{t_{f}}\left(\ell(z(t), u(t))+\phi\left(z(t), t_{f}\right)\right) d t \\
\dot{z}(t)=f(z(t), u(t)), \forall t \in\left[0, t_{f}\right] \\
z_{I_{1}}(0)=c_{1}, z_{I_{2}}\left(t_{f}\right)=c_{2} \\
a \leq C(z(t), u(t)) \leq b
\end{array}\right.
$$

where $J: \mathbb{R}^{n+m} \rightarrow \mathbb{R}, f: \mathbb{R}^{n+m} \rightarrow \mathbb{R}^{n}$ and $C: \mathbb{R}^{n+m} \rightarrow$ $\mathbb{R}^{q}$ correspond respectively to the cost function, the dynamic of the problem having a unique state trajectory and the constraints. The second equation giving the trajectory is a nonlinear system with states in $R^{n}$. In this paper, limit conditions are specified and their values are given or kept free. $t_{f}$ is also fixed. Different methods for solving the optimal control problem exist in the open literature. There are no practical theoretical limitations to using those methods that cannot be guaranteed to provide a global solution. We assume that the problem has an optimal solution with an optimal cost. In this paper, we have applied an indirect method [27-30], based on Pontryagin's principle [33-36] assessing the solutions. Inequality constraints are carried out by Pontryagin's maximum principle. We transformed the $(O C P)$ into a new unconstrained $(O C P)$ formulation that can be numerically solved. The new unconstrained $(O C P)$ is obtained by having the same system dimension. To solve $(O C P)$ problems, many methods exist in the open literature [31-36]. In this paper, we have used the optimal control technique developed in the references [16-19] which we have summarized. In depth calculation, details are given in the quoted papers. We stated that:

$$
\mathscr{H}: \mathbb{R}^{n} \times \mathbb{R}^{m} \times \mathbb{R}^{n} \times \mathbb{R}^{q} \times \mathbb{R}^{q} \longrightarrow
$$

$\mathbb{R}$ the hamiltonian of the $(O C P)$ :

$$
\begin{aligned}
\mathscr{H}(z, u, p, \lambda, \mu)= & \ell(z, u)+\phi\left(z, t_{f}\right)+p^{t} f(z, u) \\
& +\lambda^{t}(C(z, u)-a)+\mu^{t}(b-C(z, u))
\end{aligned}
$$

where $\lambda, \mu$ are the multiplicators associated to the constraints and $p$ is the costate vector [37]. We describe the optimality conditions $(O C)$ for the $(O C P)$ as:

$(O C)$

$$
\left\{\begin{array}{l}
\dot{z}(t)=f(z(t), u(t)) \\
\dot{p}(t)=-\mathscr{H}_{z}(z(t), u(t), p(t), \lambda(t), \mu(t)) \\
u(t)=\operatorname{Argmin}_{w} \mathscr{H}(z(t), w, p(t), \lambda(t), \mu(t)) \\
0 \quad=\lambda \cdot(C(z(t), u(t)-a), \quad \lambda \geq 0 \\
0 \quad=\mu \cdot(b-C(z(t), u(t)), \quad \mu \leq 0
\end{array}\right.
$$

The interior point method $[7,10,11]$, discretizing the optimality conditions of the $(O C)$, is used. $(O C)$ is transformed into a sequence of problems where the solution of optimality conditions is a solution of the $(O C)$ problem: Euler scheme discretization combined with Newton method resolution [11, 38] are used. The annex gives details of:

- the perturbation of the two last equation of $(O C)$ by a positive parameter $\varepsilon$ corresponding to the complementary conditions,

- the optimality conditions, their discretization, and how to solve the discretized problem.

\section{Numerical results}

We consider an aircraft landing by fixing initial and final flight conditions as follow:

$$
\begin{array}{lll}
0^{\circ} & \leq \alpha \leq+20^{\circ} \\
-10^{\circ} & \leq \chi \quad \leq+10^{\circ} \\
-5^{\circ} & \leq \mu & \leq+5^{\circ} \\
-10^{\circ} & \leq \phi & \leq+20^{\circ} \\
-60 \mathrm{~km} & \leq x(t) \leq 0 \mathrm{~km} \\
-10 \mathrm{~km} & \leq y(t) \leq+10 \mathrm{~km} \\
0 & \leq t & \leq+10 \mathrm{~min} \\
0^{\circ} & \leq \gamma & \leq \gamma_{\max }=\text { free } \\
85 \mathrm{~m} / \mathrm{s} & \leq V(t) \leq V_{\max }=\text { free } \\
0.2 & \leq \delta_{x}(t) \leq \delta_{x_{\max }}=\text { free } \\
125000 \mathrm{~kg} & \leq m(t) \leq m_{f}=\text { free } \\
3500 \mathrm{~m} & \leq h(t) \leq h_{f}=\text { free }
\end{array}
$$


$V_{\min }=85 \mathrm{~m} / \mathrm{s}$ represents the aircraft stall velocity. Some of these parameters are kept free. Once the processing steps and calculation efficiency are confirmed, their limit values are found and given. The data used in this optimization model are taken from an Airbus A300 of which the specifications are as follow:

- $\quad$ Type : Airbus A300 - 600

- Powerplants : Two 262.4 kN GE CF6-80C2 or $2 \times 275 \mathrm{kN}$

- Weights : max TOF $165900 \mathrm{~kg}$. Operating empty $90965 \mathrm{~kg}$

- Wing span $44.84 \mathrm{~m}$. Wing area $260 \mathrm{~m}^{2}$

- Length $53.60 \mathrm{~m}$. Height $16.54 \mathrm{~m}$

- Max speed : Mach 0.84

- Fuel max capacity : 620001

The three-dimensional analysis is useful in enhancing the reliability of the optimization model which it could be applied in automatic detection of aircraft noise. We consider $R$ the distance aircraft-observer as:

$R=\left(x-x_{o b s}\right)^{2}+\left(y-y_{o b s}\right)^{2}+h^{2}$

where $\left(x_{o b s}, y_{o b s}, 0\right)$ were the coordinates of the observer on the ground under the flight path. They were located between $10 \mathrm{~km}$ and the touchdown point under the flight path. OCP was discretized along its state $z=(V, \gamma, \chi, x, y, h)$ and control $u=\left(\alpha, \delta_{x}, \mu\right)$ variables. The discretization parameter was $N=100$ points because of the solution stability. To solve the $N_{\varepsilon}$ and NLP problems, we used the AMPL model [12, 17] and a SNOPT solver. They were chosen after numerous comparisons among other standard solvers available on the NEOS optimization platform. We used the call-by-need mechanism which memorized automatically the result of the cost function in order to speed up call-byname evaluation. This method considers a sequence of $N_{\varepsilon}$ problems (tending $\varepsilon$ to zero). The problem $N_{\varepsilon}$ is initialized by centering the state and the control. Then, we initialize the Lagrange multiplicators as follows:

$$
\lambda=\varepsilon(C(z, u)-a)^{-1}, \quad \mu=\varepsilon(b-C(z, u))^{-1}
$$

For the implementation of the penalty parameter $\varepsilon$ and computation, we used the following strategy [39]:

$\varepsilon_{k+1}=\varepsilon_{k} / a, \quad a>1$

For each iteration of the interior point method, the algorithm found an optimal solution. Varying $\varepsilon$ from 1 to $1.28 \times 10^{-5}$, the feasible error increases from $5.6 \times 10^{-12}$ to $2.1 \times 10^{-7}$, and the optimality error on the solution varies from $10^{-10}$ to $3.8 \times 10^{-9}$. The processing time is equal to $5.27 \mathrm{sec}$ (5.836 CPU). Despite feasibility errors and the time processing duration, this method provides the optimal trajectory and its flight parameters. The solution trajectory (the optimal one), the Mach number and the aircraft finesse are shown in Fig. 1.

Optimization processing confirms a stabilization of the flight. The altitude $h$ decreases with three gradual slopes. We observe a two-segment approach with an alignment on the runway axis with a slope of $3^{\circ}$ during the last approach segment. Angle of descent is stable as recommended by ICAO and aircraft certification [40-42] in favor of our method. The flight rate descent is about $1060 \mathrm{ft} / \mathrm{mn}$ which
Fig. 1 Optimal flight path, Mach number and aircraft finesse variation during the approach phase
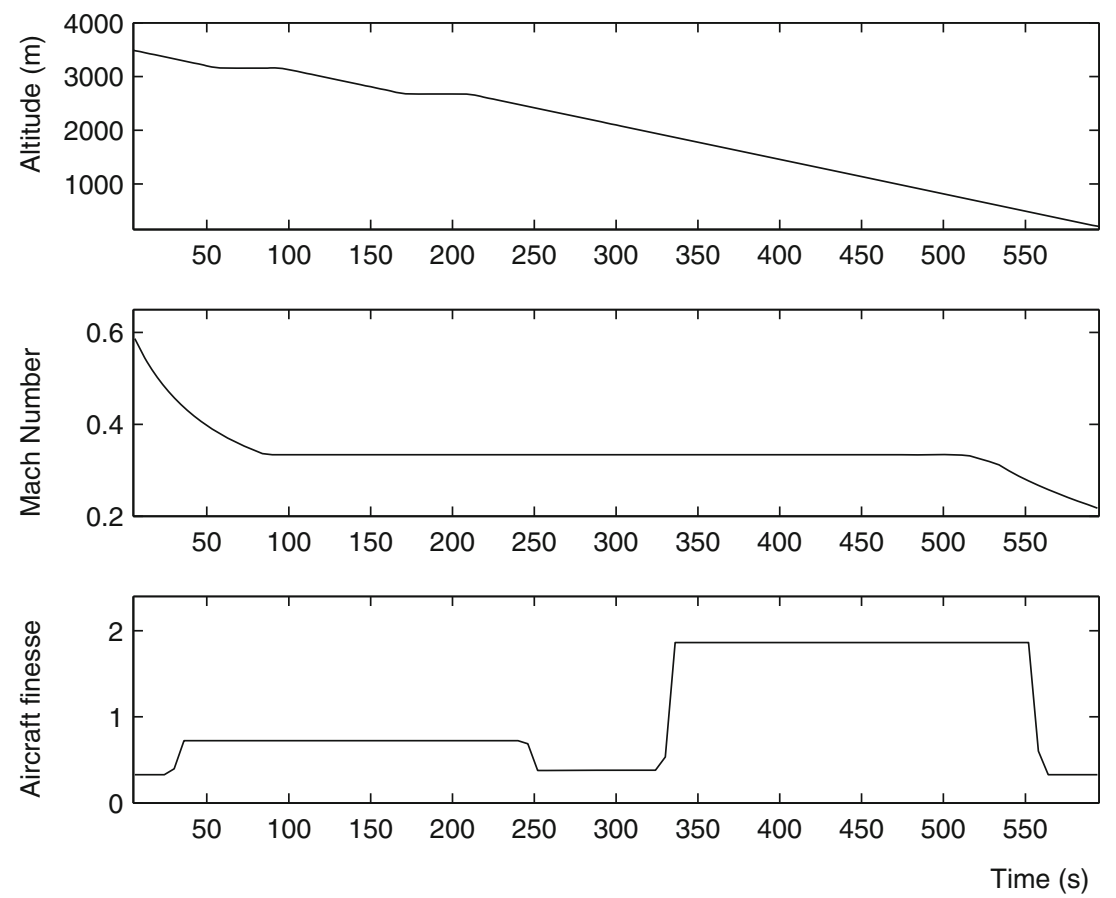
is close to the one recommended by ICAO and practiced by the airline companies $(1000 \mathrm{ft} / \mathrm{mn})$. The two segment durations obtained are respectively $39 \mathrm{sec}$ at the altitude $3150 \mathrm{~m}$ and $39 \mathrm{sec}$ at $2673 \mathrm{~m}$. This method, characterizing the continuous descent approach CDA, can be considered efficient. It should be remembered that the later can not be the fastest and shortest CDA. Nevertheless, it has the potential to reduce noise emission and fuel consumption during the approach. To conclude, the obtained optimal trajectory could be accepted into the airline community. The soft two-segment approach puts the aircraft in an appropriate envelope with margins for wind uncertainties and errors. There is no question of vortex separation and problems of intercepting a false glide-slope, given that it must be intercepted from above. With autopilot or flight director coupling, this approach would be acceptable for use in regular air carrier service. Aircraft speed, or Mach number, decreases during the first $70 \mathrm{~s}$, remains stable during almost $460 \mathrm{~s}$ (which corresponds to the whole period of the approach), and decreases during the last $70 \mathrm{~s}$ of the approach. This speed behavior is suitable for the continuous descent approach accompanied by noise and fuel consumption reduction. The aircraft finesse is bang-bang between its bounds ( 0.5 and 2$)$. At a constant speed, when the altitude decreases the thrust increases. It increases during the second flight period. Additionally, in spite of the fact that the maximum input values of the aircraft speed, the throttle setting and altitudes are kept free, the method is fast, errors are weak, and the provided calculations are exact with a correct convergence. If the guidance is satisfactory and the visual conditions are met, then CDA is considered as a managed approach. It allows avoidance of flight difficulties, in particular when the speed falls below the speed target -5 knots or rises above the speed target +10 knots, the pitch altitude becomes lower than $-5^{\circ}$ or greater than $12^{\circ}$ nose up, the bank angle becomes greater than $7^{\circ}$, and when the descent rate varies suddenly around $1000 \mathrm{ft} / \mathrm{min}$. CDA can help the flight crew to make timely and correct thrust settings, and approach path corrections if necessary. It could considerably reduce the go-arounds: pilot workload, fuel and time of flight reduction. CDA is appropriate for the aircraft stabilization in the lower part of $1000 \mathrm{ft}$ with the right thrust. Obviously when the flight is not stabilized the go-around becomes a necessity.

Figure 2 presents noise levels under the flight path versus the approach distance between $10 \mathrm{~km}$ and the touchdown point on the runway. This figure shows the evolution of noise levels given by INM under the flight path (INM curve) $[16,17]$ for standard trajectories in accordance with ICAO specifications [22, 40-42], and by the method described in this paper. During approach, noise levels increase because the aircraft altitude decreases. The tendency curve of the two methods (INM and optimization) is similar. By comparison,

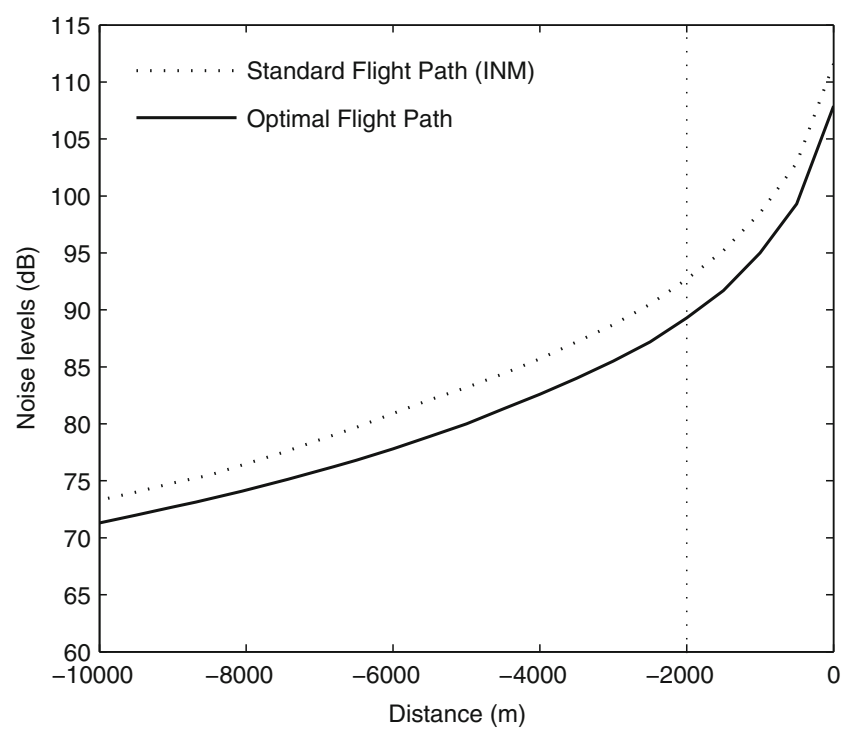

Fig. 2 Noise levels under the flight path (standard and optimal) vs. approach distance

noise levels obtained by the optimization method are lower than those obtained by INM. This method can be considered the best in terms of modeling and calculation. It is powerful and efficient. In spite of the absence of all noise models of different aircraft sources, the obtained values are close to experimental measurements, in particular at the certification point on the ground at $2 \mathrm{~km}$ under the flight path.

On the one hand, we can confirm a decrease in noise levels using the optimized flight path. At $2 \mathrm{~km}$ under the flight path, the noise level calculated by INM is equal to $93 \mathrm{~dB}$ for standard trajectories and the optimized level is $89 \mathrm{~dB}$ for the optimal trajectory. The mean noise level obtained by INM is $85 \mathrm{~dB}$ and that obtained for an optimal trajectory is $82.5 \mathrm{~dB}$. It should be noted that these calculations have taken into account the only available model of jet noise. Further research is needed including all aircraft noise sources when they become available. A $4 \mathrm{~dB}$ reduction is obtained in favor of this method compared to INM calculations at the certification point. On the other hand, comparison between noise levels corresponding to the standard trajectory obtained by INM, and the optimal trajectory given in this paper, provides changes due to the altitude of approach. Those changes are respectively equal to $4.3 \%$ and 4.5 of $\frac{J_{I N M}-J_{O p t}}{J_{I N M}}$ and $\frac{J_{I N M}-J_{O p t}}{J_{O p t}}$.

In addition, we carried out experiments under the aircraft flight path where we recorded noise levels during one year. Comparisons between calculations and measurements at the certification point $(2 \mathrm{~km})$ under the flight path have been performed. The aim of the following work is to validate, by INM, the calculations undertaken.

The measurements of noise, generated by aircraft at approach, were carried out under the flight path close to 
Lyon Saint-Exupery International Airport (France) for one year according to the annex 16 of the ICAO convention. Noise signals were recorded on line. Locations for recording aircraft noise in flight are surrounded by flat terrain having no excessive sound absorption characteristics (grass fields cut). No obstructions exist which could influence the sound field from the aircraft within a conical space above the point on the ground vertically below the microphone. The cone being defined by an axis is normal to the ground and is halfangle $\left(80^{\circ}\right)$ from this axis. The type of aircraft was recorded; it has been collected in real-time for each flight. In this paper, we present data recorded under flight path at a lateral distance of $2 \mathrm{~km}$ to the touchdown point. This measurement point corresponds to the certification point of the aircraft. The stability of atmospheric conditions was checked and timetabled. The following numbers show their fluctuations in the intervals where stability criteria are met during measurements. We summarize below meteorological parameters provided by Meteo France:

- Wind speed (m/s): $1-3$

- Average temperature $\left({ }^{\circ} \mathrm{C}\right): 15-35$

- Cloudiness (octas): $0-2$

- Humidity (\%): $35-50$

- Global radiation $\left(\mathrm{J} / \mathrm{cm}^{3}\right): 240-290$

A SIP 95 sound level meter was used to record acoustic data. The measurement system is inspected every two years and approved by the French National Laboratory for testing in accordance with international standards. The microphone is positioned at $4 \mathrm{~m}$ above the ground to comply with the requirement of the free field condition. The ground is flat and consists of grass shorter without brush, wood or obstacles. Two calibrations are performed every day. The free-field sensitivity level of the microphone and preamplifier in the reference direction, at frequencies over at least the range of one-third-octave nominal mid-band frequencies from $50 \mathrm{~Hz}$ to $10 \mathrm{kHz}$ inclusive, is within $1.0 \mathrm{~dB}$ of that at the calibration check frequency, and within 2.0 $\mathrm{dB}$ for nominal mid-band frequencies of $6.3 \mathrm{kHz}, 8 \mathrm{kHz}$ and $10 \mathrm{kHz}$. The output of the analysis system consists of one-third octave band sound pressure levels as a function of time, obtained by processing the noise signals with the following characteristics: a set of 24 one-third octave band filters [50 Hz-10 kHz]; response and averaging properties in which the output from any one-third octave filter band is squared, averaged and displayed or stored as timeaveraged sound pressure levels; the interval between successive sound pressure level samples is $500 \mathrm{~ms} 5 \mathrm{~ms}$ for spectral analysis with or without slow time-weighting. Ambient noise, including both an acoustical background and electrical noise of the measurement system was recorded for 10 minutes a day with the system gain set at the levels used for the aircraft noise measurements. The recorded aircraft noise data is acceptable according to international standards. The exclusion criteria of the recorded data are: days on which a strike took place and special weather conditions (gusty winds, stormy rainfall, atmospheric turbulence, etc.). According to the measurement specifications, we identified and retained 15460 turbojet aircraft approaching the airport in the same conditions representing $84.5 \%$ $(+20 \mathrm{~T})$ of the air traffic $(15 \%$ of the air traffic represents propeller aircraft $(3-9 \mathrm{~T}$ and $+20 \mathrm{~T})$ and $0.5 \%$ others ( $-3 \mathrm{~T}$ and 3-9 $\mathrm{T})$ ). In this paper, we analyzed the measured maximum noise levels. This is because the model described in the previous sections provide the maximum noise values.

Figure 3 presents experimental noise levels obtained at the certification point for our type of aircraft: among the 410 aircraft movements recorded per day, only one aircraft corresponding to the one chosen for this study landed in the Lyon Saint-Exupery International Airport corresponded (A300 type). Maximum noise levels of 365 aircraft are shown in the figure (one year measurements). Maximum noise levels vary from 91.9 to 100.7. These variations depend certainly on the atmospheric conditions, the aircraft loads or the effective weight in approach (unavailable information), and the type of the procedure initiated by the pilots. The average experimental value is $95.7 \mathrm{~dB}$ whereas the mean levels obtained by INM and optimization processing at $2 \mathrm{~km}$ under the flight path are respectively of $93.7 \mathrm{~dB}$ et $89.3 \mathrm{~dB}$.

On the one hand, the value obtained by INM, at $2 \mathrm{~km}$ under the flight path is in the interval of the observed values. On the other hand, the optimal value is largely below the measured and INM values. Thus, the optimal flight path

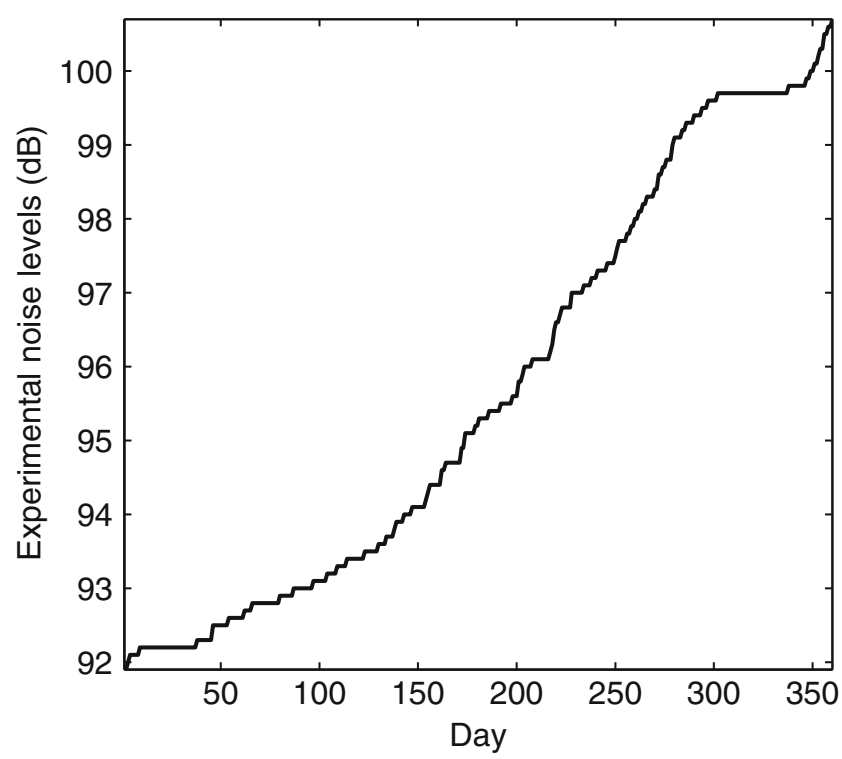

Fig. 3 Maximum noise levels measured at $2 \mathrm{~km}$ under the flight path for one year 
favors a noise level reduction between $-11.7 \mathrm{~dB}$ and -3 $\mathrm{dB}$. This significant noise reduction could be regarded as over-estimated because the other models of fuselage (aerodynamic) are not introduced in the optimization model. Nevertheless, levels obtained by the optimization and modeling processes are promising because the calculated values are validated by the experiment at the certification point of the approach. Because the engine performance could be described in several ways, three calculation methods of fuel consumption have been carried out using models by Houacine et al. formulation developed by Benson [17, 44], Mattingly [21] and Roux [43] respectively. We have calculated the most useful parameter of the engine which is represented by the fuel consumption. For turbojets, it is usually expressed as the specific fuel consumption SFC. It is defined as the weight of the fuel burned per time unit ( $\mathrm{kg} / \mathrm{second}$ in SI units). Using the optimization method given in this paper, we have also calculated the ratio of aircraft thrust on the aircraft static thrust versus speed of turbojet and by varying bypass ratio (BPR is defined as the ratio between the mass flow rate of input air to the mass flow rate passing through the engine core which is involved in combustion to produce and to increase mechanical energy) for showing benefits of optimization in terms of thrust reduction during the landing. We have used the Mattingly and Roux plan model of SFC (PIM curve in Fig. 4) and Houacine et al. (non optimal curve shown in Fig. 4). In this paper, calculation of fuel consumption is introduced as a second term of the objective function. We have used the instantaneous fuel consumption representing the fuel burnt during the approach period. Because the initial aircraft mass $m_{t_{0}}$ is assumed constant and because the weight of the aircraft at the moment $t$ is $\left(m_{t_{0}}-m_{t}\right)$, we can write the

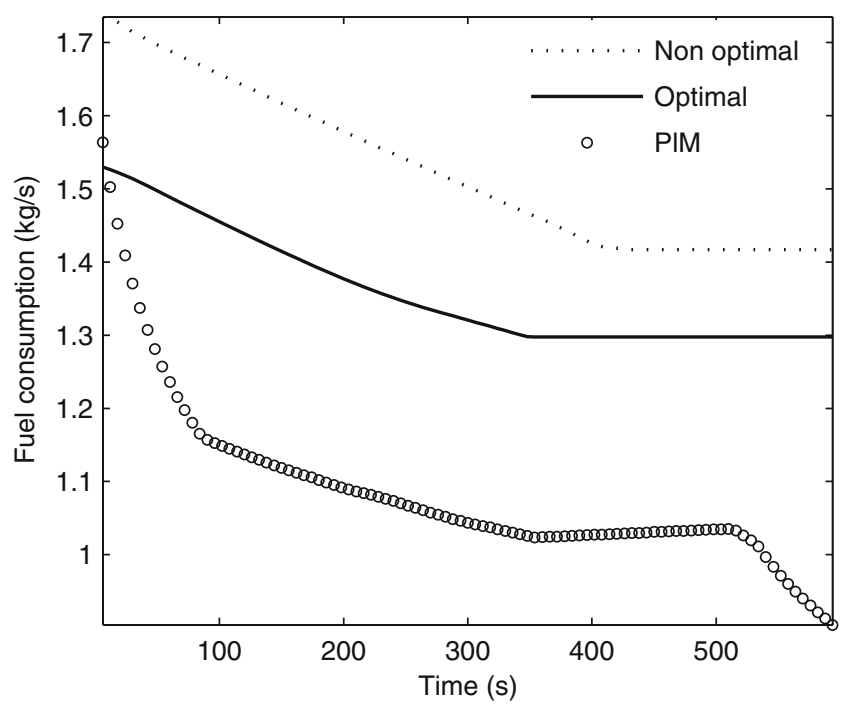

Fig. 4 Fuel consumption $(\mathrm{kg} / \mathrm{s})$ during the approach for three different models second term of the objective function by the following expression:

$$
\min \left\{\phi\left(z_{t_{f}}, t_{f}\right)\right\}=\min \left(-m_{t_{f}}\right)
$$

where $\left(m_{t_{0}}-m_{t}\right)$ is the final aircraft mass. Figure 4 presents a comparison result of the fuel consumption calculated using the three methods described.

The three used models confirmed a decrease of the fuel consumption during the approach with a variable stage. Regarding the model of the plan by Mattingly and Roux, fuel consumption decreased with sudden changes, in particular during the beginning and the end of the approach. FC is underestimated. The two other models have no sudden change in the FC evolution. The Benson model seems suitable even if it tends to over-estimate. The model of optimization provided fuel consumption values between the two model limits. What can be retained is the fact that the optimal trajectory would consume less than the standard trajectory. In addition, comparison between models provided $10 \%$ and $20 \%$ of $\frac{F C_{\text {Nonoptimal }}-F C_{O p t}}{J_{\text {Nonoptimal }}}$ and $\frac{F C_{O p t}-F C_{P I M}}{J_{O p t}}$. This fuel consumption could be in favor of optimal flight path. Models describing engine parameters during flight operations in different conditions are not often published in the open literature.

Indeed, in-flight engine database is not provided by engine manufacturers or airline companies. Data on FC and thrust versus aircraft Mach, altitude, throttle settings and power extraction can be correctly modeled because the physics behind it is well established. The output power of the turbojet is quite constant with changes in speed, whereas turbojet provides a constant thrust with speed. Modern aircraft are equipped with engines where the net thrust is reduced with the increase of the speed and the altitude.

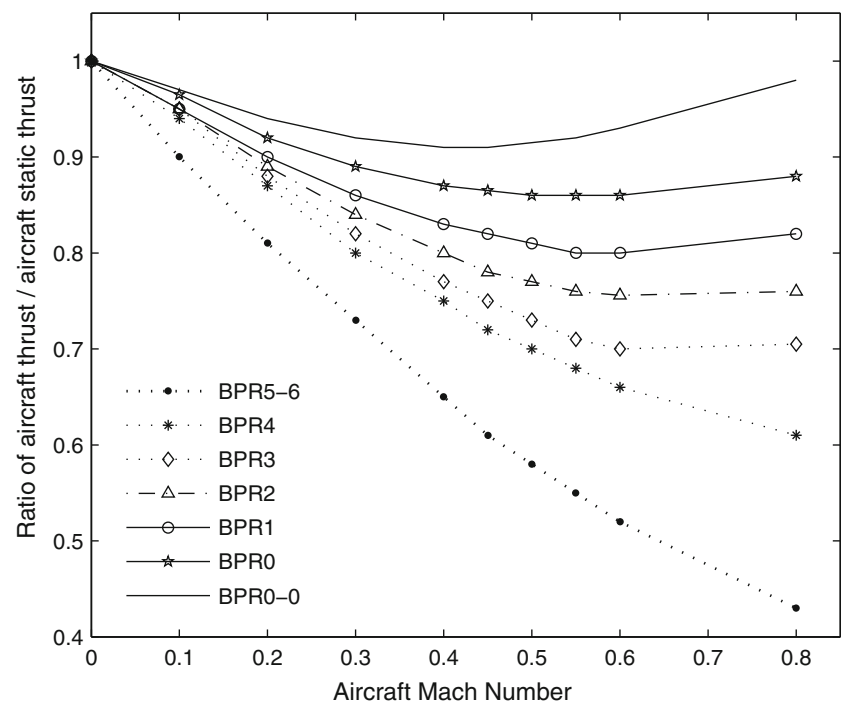

Fig. 5 Thrust behavior versus aircraft Mach number with bypass ratio variation 
Figure 5 presents a complementary analysis which can explain the behavioral characteristics of the fuel consumption that is previously given. It gives, in particular, the thrust behavior versus aircraft Mach number with bypass ratio variation. The bypass ratio is varying between 0 and 6 . The propulsive efficiency is confirmed. For any available engine energy, dynamical thrust is optimized because of the bypass ratio optimization. This is interpreted by the relationships in an action-reaction propulsion system. Thus, for a high bypass, the thrust is generally derived from the duct of the fan, rather than from combustion gases expanding in the engine nozzle. A high bypass ratio provides a lower thrust specific fuel consumption (grams/sec fuel per unit of thrust).

Low-bypass system is more fuel efficient and is much quieter. Low bypass ratios do not tend to be favored for civilian aircraft because of the compromise between improved fuel economy and the requirements of the performance of the aircraft in terms of flight paths and its stability. The behavior of thrust is common and does not present any sharp nor sudden fluctuations. Constraints on engine parameters can easily impact on the engine pressure and its temperature, and could be critical at some operating points. This is why fuel consumption has a significant role and is associated with engine performance. This association has the advantage of giving lower thrust values for extending engine life and reducing loads. Engine performance of modern engines depends on requirements of accessories, engine size, inlet and duct design, air conditioning system compressor, hydraulic pumps power, alternators power, inlet and exhaust duct losses, ... Because of the technological development of these engines, thrust is reduced; but fuel consumption can increase. Optimization of the flight path associated with new technological developments contribute significantly to the reduction of fuel consumption. Environmental impact and fuel consumption are reduced by the use of optimization process for departures and arrivals. Operation management could be also improved by using optimized flight path. In addition, these flight path parameters can be integrated in the flight management system of the aircraft, and used in the autopilot system. This is a promising objective of this research. This bypass ratio study could contribute to provide numerical results and data for the future generation engines which will have a higher bypass ratio. In association with passive control noise systems, the primary advantage of this contribution should be engine efficiency analysis and weight savings. The second advantage, linked to the first, is the drag reduction. The third advantage, related to the increase of the bypass ratio, helps airline manufacturers to suggest the best arrangement of nacelle components to facilitate convenient removal and replacement of engines without subjecting nacelle to high stresses. To conclude, optimization of flight path induces fuel consumption savings, noise reduction and improvement of the engines-BPR knowledge.

\section{Conclusion}

Optimization model is expected to replace empirical models for well-established applications such as predicting noise contours around airports and fuel savings. Despite numerical complexity, feasibility errors and time processing duration, this paper suggests a method which provides optimal trajectory and its parameters reducing noise and fuel consumption. In this technical paper, we applied the best numerical method associated with an adequate algorithm we have developed for solving aircraft trajectory optimization problem. It has taken into account jet noise source, fuel consumption, aircraft constraints and its operational extreme limits. Additionally, in spite of the fact that the maximum input values of the aircraft speed, the throttle setting and altitudes are kept free, the method gives reliable results. It is fast, errors are weak, and calculations are exact with a suitable convergence time. This new original approach contributes to improve scientific knowledge in the field of environmental impact reduction of aircraft.

The altitude decreases with three gradual slopes with two segments. Aircraft alignment on the runway axis is performed with a slope of $3^{\circ}$ during the last approach segment. Angle of descent is stable as recommended by ICAO and aircraft certification. The flight rate descent is about 1060 $\mathrm{ft} / \mathrm{mn}$ which is close to the one recommended by ICAO and practiced by the airline companies.

This method characterizes the continuous descent approach (CDA) which has the potential to reduce noise emission and fuel consumption during the approach. The obtained optimal trajectory could be accepted into the airline community. The soft two-segment approach puts the aircraft in an appropriate envelope with margins for wind uncertainties and errors. With autopilot or flight director coupling, this numerical method could be acceptable for use in regular air carrier service.

In addition, Mach number decreases during a short period of the flight and remains stable during the whole approach. The aircraft finesse is bang-bang between its bounds. At a constant speed, when the altitude decreases, the thrust increases. It increases during the second flight period. If the guidance is satisfactory and the visual conditions are met, CDA is considered as a managed approach.

Evolution of the obtained noise levels under the flight path, given by INM for standard trajectories in accordance with ICAO specifications, is compared with the optimized values behavior. By comparison, optimized noise levels are lower than those obtained by INM. In spite of the absence of all noise models of different aircraft sources, calculated 
values are close to experimental measurements. A decrease in noise levels can be confirmed when practicing the optimized flight path. A $4 \mathrm{~dB}$ reduction is obtained in favor of the optimization method.

In addition, we carried out experiments under the aircraft flight path to validate benefits of flight path optimization in term of noise reduction. Measurements of noise were performed under the flight path close to Saint-Exupery Lyon International Airport during one year. We identified 15460 turbojet aircraft executing approaches of the airport in the same conditions: $84.5 \%(+20 \mathrm{~T})$ of the air traffic are turbojet aircraft, $15 \%$ are propeller aircraft $(3-9 \mathrm{~T}$ and +20 T) and $0.5 \%$ others (-3 T and 3-9 T). 360 aircraft are A300 or equivalent. Maximum noise levels vary from 91.9 $\mathrm{dB}$ to $100.7 \mathrm{~dB}$. These variations depend certainly on the atmospheric conditions, the aircraft loads, and the procedure variations initiated by the pilots. At the certification point, average experimental values is $95.7 \mathrm{~dB}$ whereas the mean levels obtained by INM and the optimization method are respectively $93.7 \mathrm{~dB}$ and $89.3 \mathrm{~dB}$. Optimal mean value is largely below the measured and INM values. Thus, comparisons show that optimal flight path favors noise level reduction. This significant reduction could be regarded as over-estimated because the other noise models of fuselage are not introduced in the optimization model. Nevertheless, the optimization method is promising.

Engine performance is assessed by three calculation methods. Using the optimization method, we have calculated the ratio of aircraft thrust on the aircraft static thrust versus speed and by varying bypass ratio. Calculation of the fuel consumption is introduced as a second term of the cost function. In the open literature, specific fuel consumption have underestimated or overestimated the burned fuel, or shown sudden changes. Optimization model provides fuel consumption values between the two first model limits. Optimal trajectory consumes less than the standard trajectory. Comparison between models provided $10 \%$ to $20 \%$ of fuel savings in favor of the optimized flight path.

The behavior of the thrust has no sudden fluctuations, confirming the propulsive efficiency. For available engine energy, dynamical thrust is optimized because of the bypass ratio improvement. This is interpreted by the relationships in an action-reaction propulsion system. Constraints on engine parameters easily impact the engine pressure, its temperature and could be critical at some operating points. This is why analysis of fuel consumption has a significant role because it is associated with engine performance. This association has the advantage to lower thrust values for extending engine life and reducing loads.

To conclude, optimization of flight path associated with new technological developments should contribute significantly to the reduction of noise and fuel consumption. In particular, association with passive control noise systems, one of the advantages of this contribution should be engine efficiency analysis and weight savings. Environmental impacts and fuel consumption are reduced by trajectory optimization of aircraft during arrivals. Optimized flight path can be integrated in the flight management system and can be used in the autopilot system. This is a promising objective of this research. Further research is needed to include airframe noise sources, and air-brake systems.

Issues and alternatives Aviation industry is crucial to world trade. Its global economic impact is equivalent to more than 7.5 per cent of world GDP. It directly employs more than 5.5 million people world-wide. Besides economic impacts, aviation plays a pivotal role in connecting communities. The low cost flights have supported a strong demand growth. Neither reduction at source using quieter aircraft nor operational restrictions (noise action plans, direct government regulation or voluntary airport initiatives) are delivering satisfactory mitigation. ICAO Balanced Approach to aircraft noise control consists of identifying noise problems at an airport, then analyzing various measures. Long-term measures must force the solutions at regional level: reduction of noise at source and certification; phase-out of noncertificated airplanes; noise charges; and land-use planning and management. Short-term measure must facilitate the solutions at local level, like noise abatement procedures and mitigation of aircraft operation. It is a major challenge for the future of air transport in the context of economic development linked to compliance with the conditions of people living near airports. The land use planning element of ICAO's balanced approach is difficult to implement.

The use of advanced aircraft, optimized flight paths and improved airspace management offer the most immediate ways to mitigate aviation's environmental impact. However, against growing demand for air transportation system these efforts alone are unlikely to be sufficient for a significant impact reduction in the long term. This paper gives a new change to flight path approach which is characterized as a noise abatement technique. Pilots can descend at the rate best suited to the achievement of continuous descent, with the objective to join the glide path at the appropriate height. Rather than deploying flaps and descending through a given number of flight levels, the aircraft flight a continuous steady descent at a fixed angle. It should be remembered that $\mathrm{P}$ (recision)-RNAV in Europe, defined as operations which satisfy a required track keeping accuracy of $1 \mathrm{NM}$ for at least $95 \%$ of the flight time, is not in contradiction with the optimized flight path developed in this paper.

The main benefit of the optimized flight paths are that they avoid flaps deployment and thrust changes. The aircraft may also be higher at points along the approach path. Because of little few gear and flap changes, aircraft stay higher along the descent segments contributing to noise 
and fuel reduction. Because of environment benefits, the use of the optimized flight path as a noise management tool is advocated by regulators worldwide, from the ICAO to national governments. It also helps to reduce fuel consumption allowing an added value for airlines. Optimized flight path is advised, promoted, and incorporated in a voluntary code of practice compiled by airlines, air traffic control, airport authorities and the transport departments of the European countries. This is a major action intending to emphasize measures that can increase flight number and avoiding air traffic saturation. Because of the growth in traffic, more flights can join the final approach without conflict and with less community annoyance. Thus, a compromise should be found between environmental acceptability, the lower cost of design, development, production and exploitation, and increasing the operational capacity of the airspace.

Policy recommendations It is clear that neither quieter aircraft nor operational restrictions are satisfactory. Nevertheless, optimized flight paths have potential environmental and economical benefits compared to the present day approach procedures including:

- higher altitude during a large part of the approach,

- lower power settings with clean aircraft configuration,

- more flexibility in definition of approach path geometry, enabling the procedure designer to define approach paths away from residential area

- additional advantage of the safety issue by reducing third party risk.

There is sufficient evidence in this paper to support further assessment of optimized flight paths which provide flexibility where problems of flight concentration exist. Annoyance is strongly influenced by the number of aircraft. Noise policies and measures, taking into account the traffic increase, have contributed to the communities living around airports. Limiting the number of the population impacted by aircraft noise can improve the quality of life for them and reduce the effect of concentrated flight paths. Noise problem has evolved over the last decades and become sensitive because it is now associated to air pollution problems affecting health population. Air traffic increase, new aircraft technology and airspace management associated to local action by airport operators makes the environmental policy reliable. Because of air traffic increase, the difficulty in modifying flight paths location and protecting the population have all added to the complexity of this environmental problem. A new policy needs to consider these issues, and a perpetual revision should be a high priority. We recommend that the policy needs to include a general environmental duty in performing its operational actions. Airport authorities and the policy need to have a sharp regard for environmental factors alongside the safety and consumer objectives. Failure to do this will leave airport authorities to explain what is reasonable when taking decisions on matters such as airspace changes.

The preliminary question is what is the best way to achieve environment objectives associating technological development, the renewal of aircraft, implementation of aircraft optimized procedures, airspace management, ... In addition to new environmental finding allowing to improve environmental impacts of aircraft, scientific communities need to provide specific guidance relevant to air navigation functions. Air traffic authorities also need to update the existing guidance, and European governments should extend a revision of the existing noise policy. Airport operators, governmental environmental committees, airlines, air traffic managers and aircraft manufacturers should be actively engaged to initiate trials to assess the potential benefits of the combined possible solutions. Both governments and aircraft industries should address actions toward greenhouse gas emissions from aircraft and fuel saving rather than focusing on the only noise issues where emerging ideas could be developed.

Sustainability is a key issue for aviation which is united in its commitment to develop global solutions for the sustainable future of international civil aviation. With the flight paths to a sustainable future initiatives, ICAO has highlighted the capability of national authorities, aircraft manufacturers, airlines, air navigation service providers, and other stakeholders to work together to make this new flight paths possible. In addition, sustainability for airports means not only minimizing environmental impacts but also communicating about the social and economic benefits of airports and aviation. Without taking into account the social requests, airports will not be able to meet future capacity demands. The following topics have to be considered:

- Safety and security (safety, sustainable development and control capacity, airport development, automatic assistance of control, ...);

- Management and user service;

- European construction and preparation of the future (Airspace, SESAR, support the implementation of the new coming technologies of communication, navigation, monitoring, ...);

- World politics of air traffic navigation.

They are declined in four major actions to support:

1. promoting research and development into new low noise engine and airframe technologies;

2. implementing the regulatory framework agreed by the International Civil Aviation Organization (ICAO) using the balanced approach to noise management; 
3. implementing EU Directives which require periodic noise mapping;

4. the use of economic instruments which should have no limitations for long-term benefits.

\section{Annex}

Perturbing the last two equations corresponding to the complementary conditions, by a positive parameter $\varepsilon$, we obtained:

$\left(O C_{\varepsilon}\right)$

$$
\left\{\begin{array}{l}
\dot{z}(t)=f(z(t), u(t)) \\
\dot{p}(t)=-\mathscr{H}_{z}(z(t), u(t), p(t), \lambda(t), \mu(t)) \\
u(t)=\operatorname{Argmin}_{w} \mathscr{H}(z(t), w, p(t), \lambda(t), \mu(t)) \\
\mathbf{1} \varepsilon=\lambda .(C(z(t), u(t))-a), \quad \lambda \geq 0 \\
-\mathbf{1} \varepsilon=\mu .(b-C(z(t), u(t))), \quad \mu \leq 0
\end{array}\right.
$$

This is the optimality conditions of the following problem:

$\left(P_{\varepsilon}\right)\left\{\begin{array}{l}\min \int_{0}^{t_{f}}\left(\ell_{\varepsilon}(z(t), u(t))+\phi_{\varepsilon}\left(z(t), t_{f}\right)\right) d t \\ \dot{z}(t)=f(z(t), u(t)), \quad t \in\left[0, t_{f}\right]\end{array}\right.$

$\ell_{\varepsilon}$ is the logarithmic barrier of $\left(P_{\varepsilon}\right)$ :

$$
\begin{aligned}
\ell_{\varepsilon}(z, u)+\phi_{\varepsilon}\left(z, t_{f}\right)= & \ell(z, u)+\phi\left(z, t_{f}\right) \\
& -\varepsilon \sum_{i}\left[\log \left(C_{i}(z, u)-a_{i}\right)\right. \\
& \left.+\log \left(b_{i}-C_{i}(z, u)\right)\right] \\
& -\varepsilon D(z)
\end{aligned}
$$

To solve $(O C)$, we solved a sequence of problems $\left(O C_{\varepsilon}\right)$ by tending $\varepsilon$ to zero. When $\varepsilon$ decreases to 0 , the solution of optimal conditions $\left(O C_{\varepsilon}\right)$ is a solution of $(O C)$. Discretization of the continuous optimal conditions provided a set of non-linear equations, which is solved for the discretized control, state and costate vectors using the Newton method [37]. Euler schema provided for $\left(O C_{\varepsilon}\right)$ the following system:

$$
\left\{\begin{array}{l}
z_{k+1}=z_{k}+\mathrm{h} f\left(u_{k}, z_{k}\right), \quad k=0, \ldots, N-1 \\
p_{k+1}=p_{k}-\mathrm{h} \mathscr{H}_{z}\left(z_{k}, u_{k}, p_{k}, \lambda_{k}, \mu_{k}\right), \quad k=0, \ldots, N-1 \\
0 \quad=\mathscr{H}_{u}\left(u_{k}, z_{k}, p_{k}, \lambda_{k}, \mu_{k}\right), \quad k=0, \ldots, N \\
\mathbf{1} \varepsilon=\lambda_{k} \cdot\left(C\left(z_{k}, u_{k}\right)-a\right), \quad \lambda_{k} \geq 0, \quad k=0, \ldots, N \\
-\mathbf{1} \varepsilon=\mu_{k} \cdot\left(b-C\left(z_{k}, u_{k}\right)\right), \quad \mu_{k} \leq 0, \quad k=0, \ldots, N
\end{array}\right.
$$

We obtained a set of equations which are solved under the boundary constraints corresponding to the multiplicators:

$$
\left(N_{\varepsilon}\right) \quad\left\{\begin{array}{l}
F_{\varepsilon}(X)=0 \\
\lambda_{k} \geq 0 \\
\mu_{k} \leq 0
\end{array}\right.
$$

$F_{\varepsilon}$ is the set of optimal conditions, and $X=\left(z_{k}, u_{k}\right.$, $p_{k}, \lambda_{k}, \mu_{k}$ ) the variable vector. (OCP) is then successfully solved for decreasing $\varepsilon$ with a non-growth of the cost.

Open Access This article is distributed under the terms of the Creative Commons Attribution License which permits any use, distribution and reproduction in any medium, provided the original author(s) and source are credited.

\section{References}

1. Lambert J (2008) Perception and attitudes to transportation noise in France: a national survey, The 9th Congress of the ICBEN, Mashantucket

2. Kurniawan JS, Khardi S (2011) Comparison of methodologies estimating emissions of aircraft pollutants. Environmental impact assessment around airports. Environ Impact Assess Rev 31:240 252

3. Bellman R (1957) Dynamic programming. Princeton University Press, New Jersey

4. Pontryagin L, Boltyansky V, Gamkrelidze V, Mischenko E (1962) Mathematical theory of optimal processes. Wiley-Interscience, New York

5. Stone JR, Groesbeck DE, Zola CL (1981) An improved prediction method for noise generated by conventional profil coaxial jets. National Aeronautics and Space Administration. Report NASATM-82712, AIAA-1991

6. Hubbard HH (1995) Aeroacoustics of flight vehicles. Theory and Practice. Volume Noise sources, Published for the Acoustical Society of America through the American Institute of Physics

7. Wright S (1993) Interior-point methods for optimal control of discrete-time systems. J Optim Theory Appls 77:161-187

8. Wright S (1997) Primal-dual interior-point methods. Society for Industrial and Applied Mathematics SIAM, Philadelphia

9. Bonnans JF, Gilbert JC, Lemarechal C, Sagastizabal C (1997) Optimisation Numrique. Aspects thoriques. Mathmatiques et Applications. Springer-Verlag

10. Weiser M (2005) Interior-point methods in function space. J SIAM Control Optim 44:1766-1786

11. Berend N, Bonnans F, Haddou M, Varin J, Talbot C (2005) An interior-point approach to trajectory optimization. INRIA Report, N. 5613

12. AMPL A modeling language for mathematical programming. http://www.29.com

13. Gill P, Murray W, Saunders M, SNOPT A large-scale smooth optimization problems having linear or nonlinear objectives and constraints. http://www-neos.mcs.anl.gov/neos/solvers

14. Waltz RA (2004) KINITRO user's manual version 4.0. Ziena Optimization Inc

15. Byrd RH, Nocedal J, Waltz RA, KNITRO (2006) An integrated package for nonlinear optimization. http://www-neos.mcs. anl.gov/neos/solvers

16. Khardi S, Abdallah L, Konovalova O, Houacine H (2010) Optimal approach minimizing aircraft noise and fuel consumption. Acta Acustica United with Acustica 96:68-75

17. Houacine M, Khardi S (2010) A gauss pseudospectral method for less noise and fuel consumption of aircraft operations. J Aircr 6:2152-2158

18. Abdallah L, Haddou M, Khardi S (2010) Optimization of operational aircraft parameters reducing noise emission. Appl Math Sci 4(9-12):515-535

19. Khardi S, Nahayo F, Haddou M (2011) The trust region esquential quadratic programming method applied to two-aircraft 
acoustic optimal control problem. Appl Math Sci 5(40):19531976

20. Boiffier J (1998) The dynamics of flight. John Wiley and Sons

21. Mattingly JD (1996) Elements of gas turbine propulsion. McGraw-Hill International Editions

22. Zaporozhets OI, Tokarev VI (1998) Predicted flight procedures for minimum noise impact. Appl Acoust 55(2):129-143

23. Zaporozhets OI, Tokarev VI (1998) Aircraft noise modelling for environmental assessment around airports. Appl Acoust 55(2):99_ 127

24. Tam CKW (1995) Computational aeroacoustics. Issues and methods. AIAA J 33:1788-1796

25. Tam CKW (2001) Noise from high speed jets. In: Anthoine J, Schram C (eds) VKI lecture series on advanced in aeroacoustics. Lecture Series 2001-2002, pp 1-34

26. Zaporozhets OI, Khardi S (2004) Optimisation of aircraft flight trajectories as a basis for noise abatement procedures around the airports. Theoretical considerations and acoustical applications, INRETS Report, vol 257

27. Dreyfus SE, Averill M (1977) The art and theory of dynamic programming. Academic Press, ISBN 978-0122218606

28. Iofe AD, Tikhomirov VM (1979) Theory of extremal problems. North-Holland

29. Kamien M, Schwartz N (1981) Dynamic optimization. NorthHolland

30. Bertsekas DP (2000) Dynamic programming and optimal control. Athena Scientific, ISBN 1-886529-09-4, 2nd ed.

31. Nocedal J, Wright S (2006) Numerical optimization. Springer, New York

32. Graichen K, Kugi A, Petit N, Chaplais F (2010) Handling constraintes in optimal control with saturation functions and system extension. Syst Control Lett 59:671-679
33. Pontryagin LS, Boltyansky VG, Gamkrelidze RV, Mishchenko EF (1964) The mathematical theory of optimal processes. Pergamon Press LTD

34. Kirk DE (1970) Optimal control theory, an introduction. Prentice Hall

35. Gramkelidze RV (1978) Principles of optimal control theory. Plenum Press

36. Ross IM (2009) A primer on pontryagin's principle in optimal control. Collegiate Publishers

37. Seywald H, Kumar R (1996) Method for automatic costate calculation. AIAA J Guid Control Dyn 19:1252-1261

38. Hildebrand FB (1987) Introduction to numerical analysis. Dover, reprint Chapt. 8

39. Dussault JP, Elafia A (1999) On the superlinear convergence order of the logarithmic barrier algorithm, mathematics and statistics. Comput Optim Appl 19(1):31-53

40. Erkelens LJJ (2000) Research into new noise abatement procedure for the 21st century. AIAA Guidance, Navigation and Control Conference, Reston

41. CAEP (2007) Generic presentation on the bamanced approach. Committee on Aviation Environmental Protection. ICAO, CAEP/7-WP/16, 7th meeting, Montreal, pp 5-16

42. ICAO (2007) Review of noise abatement procedure. Research and development and implementation results. Discussion of survey results, Preliminary edition, $\mathrm{p} 29$

43. Roux E (2006) Pour une approche analytique de la dynamique du Vol. Modles Moteur. Racteurs double flux civils et racteurs militaires faible taux de dilution avec PC, Ph.D.Thesis. INSA Rouen - SupAero - ONERA, vol 1, p 280

44. Benson TJ (1995) An interactive educational tool for turbojet engine. Tech. rep., NASA Lewis Research Center, Cleveland 